\title{
A FURTHER CHARACTERIZATION OF A PROJECTIVE SPECIAL LINEAR GROUP
}

\author{
SAAD ADNAN
}

(Received 17 February 1976)

Communicated by M. F. Newman

\begin{abstract}
In this paper we present a characterization of $\operatorname{PSL}(2.7)$ by a condition different from that given in our previous paper.
\end{abstract}

\section{Introduction}

In the first place we shall fix our notation. Let $G$ be a finite group and $\pi(G)$ the set of primes each dividing the order of $G$. Then we denote by $\tau(G)$,

$\tau(G)=\{p \in \pi(G) \mid[G: M]$ is a power of $p$, where $M$ is a subgroup of $G\}$ and by $\psi(G)$, the set of pairs

$$
\psi(G)=\{(M, p)|p \in \tau(G), p|[G: M] \text { for a maximal subgroup } M \text { of } G\} .
$$

We shall be using the following hypothesis:

(*) (i) $G$ is simple

(ii) Every maximal subgroup of $G$ has index a power of a prime.

In Adnan (1976), we have been able to show that if the group $G$ satisfies hypothesis $\left(^{*}\right)$ together with the condition

(A) For every $(M, p) \in \psi, M$ is $q$-soluble for some $q \in \tau-\{p\}$, then $G \simeq \operatorname{PSL}(2,7)$.

In this paper we replace condition (A) by another condition which is easier to work with, and show that in presence of hypothesis $\left(^{*}\right)$, our new condition implies condition (A) and as such $G \simeq \operatorname{PSL}(2,7)$.

Next we proceed to state our main theorem.

MAIN THEOREM. Let $G$ be a finite group satisfying $\left(^{*}\right)$. Then $G \simeq \operatorname{PSL}(2,7)$ if the following condition $(B)$ holds. 
(B) There are primes $p, q \in \tau, p \neq q$, such that for every $(M, p),(N, q) \in \psi$, $M \cap N$ is disjoint from its conjugates.

\section{Preparatory lemmas}

Lemma 1. (Non-simplicity condition). Let $G$ be a finite group and $H$ and $K$ be subgroups of $G$ with

$$
[G: H]=p^{i} \quad[G: K]=q^{i}
$$

$p, q$ being distinct primes of $\pi(G)$. If $H \cap K$ contains a unique involution $x$, and if $C_{G}(x) \subseteq N_{G}(H \cap K)$, then $G$ is not simple.

Proof. Set $Y=H \cap K$. If $q \neq 2 \neq p$, then $Y$ contains an $S_{2}$-subgroup $T$ of $G$. Since $Y$ contains a unique involution, it follows that $T$ is either cyclic or generalized quaternion and so $G$ is not simple by p. 373 of [2]. Thus we may assume that $q=2$.

Let $B=O_{2}(Y)$. Then $B \neq 1$ by hypothesis. Moreover, if $S$ is an $S_{2}$ subgroup of $H$, then $H=Y S$. Since $Y \cap S$ is an $S_{2}$-subgroup of $Y$, we have $B \subseteq S$. We conclude that $O_{2}(H) \neq 1$.

Now let $t$ be an involution such that $t \in Z \cap O_{2}(H)$, where $Z=Z(S)$. Since $Z \subseteq C_{G}(x) \subseteq N_{G}(Y), t$ normalizes $Y$. If $r$ is an odd prime dividing $|Y|$ and $R$ is an $S_{r}$-subgroup of $Y$, then $[R, t] \subseteq[Y, t] \subseteq Y \cap O_{2}(H)$. Let $z$ be any non-identity element of $R$. Then $[z, t] \in O_{2}(H)$ and since $t \in Z$, $t$ centralises $O_{2}(H)$, and hence $t$ centralises $t^{z}$. Hence $[z, t]=t^{2} t$ is an involution in $Y$ or 1 . By hypothesis $[z, t] \in\langle x\rangle$. Similarly $\left[z^{2}, t\right] \in\langle x\rangle$ and we conclude that $[z, t]=$ 1. Therefore $t$ centralises $R$ and thus $Y$. Since $t \in Z$, we have $Y, S \subseteq C_{G}(t)$ i.e. $H \subseteq C_{G}(t)$. Thus $\left[G: C_{G}(t)\right]$ is a power of $p$ and so $G$ is not simple by p. 131 of [2].

LEMMA 2 (Solubility Criterion). If $G$ is a finite group expressible in the form $G=H Q$ where $H$ is a regular group of automorphisms of some $p$-group, having no quaternion subgroups and $Q$ is a $q$-subgroup of $G$ for some prime $q \in \pi(G)$, then $G$ is soluble.

Proof. Since $H$ contains no quaternion subgroups, we deduce that $H$ is metacyclic (by [2] p. 258). If $q \in \pi(H)$, then we write $G=K Q$, where $K$ is a Hall $q^{\prime}$-subgroup of $H$ and $Q$, a Sylow $q$-subgroup of $G$. Thus we may assume without loss of generality that $H$ is a $q^{\prime}$-subgroup of $G$.

We proceed now by induction on $|G|$. We first show that if $N$ is a non-trivial normal subgroup of $G$, then $N$ is soluble. If $Q \subseteq N$ then 
$N=(N \cap H) Q$ and by induction $N$ is soluble. If $Q \not \subset N$ then $L=H N$ is a proper subgroup of $G$. Also $Q \cap N$ is a Sylow $q$-subgroup of $N$ and hence of $L$, and so $L=H(Q \cap N)$. Again by induction $L$ and hence $N$ is soluble. In particular if $G^{\prime} \subset G$, then $G^{\prime}$ and hence $G$ is soluble. We may therefore assume that $G$ is perfect. If $O_{q}(G) \neq 1$, then clearly $\bar{G}=G / O_{q}(G)$ satisfies the hypothesis of the lemma and so $\bar{G}$ and hence $G$ is soluble. On the other hand if $O_{q}(G) \neq 1$, then let $r$ be the largest prime dividing $O_{q}(G)$ and $D$ be an $S_{r}$-subgroup of $O_{q}(G)$. Since $O_{q}(G) \subseteq H, O_{q}(G)$ is metacyclic and so $D \triangleleft O_{q}(G)$ i.e. $D \triangleleft G$. Thus $G / C_{G}(D)$ embeds into Aut $(D)$. Since $D$ is cyclic and $G$ is perfect, we have $G=C_{G}(D)$. Set $\bar{G}=G / D$. Then $1 \neq D \subseteq$ $Z(G) \cap G^{\prime}$ and so the Schur multiplier of $\bar{G}$ (see [3], p. 628) is non-trivial. However by Satz ([3], p. 642) the $r$-part of the Schur multiplier of $\bar{G}$ embeds into the Schur multiplier of $R, R$ being an $S_{r}$-subgroup of $\bar{G}$. Since $R$ is cyclic, it follows by Satz in [3] p. 643 that the Schur multiplier of $R$ is 1 , a contradiction. Therefore we may assume $O_{q}(G)=1=O_{q}(G)$ i.e. $F(G)=1$. Now let $x \in Z(H)\left(Z(H) \neq 1\right.$ by Satz in [3] p. 506). Then $\left[G: C_{G}(x)\right]$ is a power of $q$ and hence $G$ is not simple by a theorem of Burnside (cf. [2] p. 131 ). Let $N$ be a normal (non-trivial) subgroup of $G$. Since $N$ is soluble, we have $1 \neq F(N) \subseteq F(G)$, the last contradiction.

REMARK. In the proof of lemma 2 above, one can use theorem 4.4 (ii) of [2], p. 253 instead of the Schur multiplier and argue that $G$ is not perfect.

Lemma 3. Let $G$ be a finite group, then

(i) $G=M N$ for two subgroups $M$ and $N$ implies $N_{G}(M \cap N)$ is factorisable.

(ii) If $G$ satisfies $\left({ }^{*}\right)$, and if $(M, p)$ and $(N, q) \in \psi(G)$, for $p$ and $q$ distinct primes in $\tau(G)$, then $G=M N$. If $M$ is Frobenius group then $N_{G}(M \cap N) \subseteq N$. Conversely if $M \cap N$ is disjoint from its conjugates and if $N_{G}(M \cap N) \subseteq N$, then $M$ is Frobenius.

(iii) Let $G$ satisfy $\left({ }^{*}\right)$, and let $(M, p),(N, q) \in \psi(G)$ for distinct primes $p$ and $q$ in $\tau(G)$ such that $M \cap N$ is disjoint from its conjugates. If $M$ is Frobenius then $M \cap N$ is a Frobenius complement for $M$ and has odd order.

Proof. (i) Let us write $H=M \cap N$. If $g \in N_{G}(H)$, then $g=m n$, for some $m \in M$ and $n \in N$. So

$$
H^{8}=H^{m n}=H .
$$

Thus

$$
H^{m}=H^{n^{-1}} \subseteq M \cap N=H \text { i.e. } m, n \in N_{G}(H) .
$$


(ii) By (i) we may write $N_{G}(H)=\left(M \cap N_{G}(H)\right)\left(N \cap N_{G}(H)\right)$. Since $M$ s Frobenius, it follows by Thompson's theorem (cf. [2] p. 337) that $F(M) \neq 1$. 3y lemma 4 in [1] it follows that $F(M)$ is an $S_{p}$-subgroup of G. Since [M:H] s a power of $p$, we have $M \cap N_{G}(H)=H$. Thus $N_{G}(H)=N \cap N_{G}(H)$ is ;ontained in $N$. Conversely if $N_{G}(H) \subseteq N$, then as $M \cap N=H$, we conclude $\mathrm{V} \cap N_{G}(H)=H$. Further $H$ is disjoint from its conjugates and so $M$ is Frobenius.

(iii) To show $H$ is a complement for $M$, we notice that by our hypothesis $H$ is disjoint from its conjugates. Moreover since $[M: H]$ is a power of $p$ and $M$ is Frobenius with kernel an $S_{p}$-subgroup of $G$ (cf. [1], lemma 4), we have $M \cap N_{G}(H)=H$ i.e. $H$ is a complement for $M$.

To show $H$ has odd order, we notice that if $|H|$ is even, then since $H$ is a complement for $M$, by the above, $H$ contains a unique involution, say $x$. By hypothesis, $H$ is disjoint from its conjugates, and so $C_{G}(x) \subseteq N_{G}(H)$. By lemma 1, however $G$ could not be simple, thus leading to a contradiction.

To establish our main theorem we proceed to prove in two parts.

(A) Let $\mathscr{Y}=\{(M, N) \mid(M, q),(N, p) \in \psi, M$ and $N$ being non-Frobenius $\}$; then we establish first that $\mathscr{I}$ is empty.

Proof. If $\mathscr{I}$ is not empty, choose $(M, N) \in \mathscr{I}$, with $(M, q),(N, p) \in \psi$, such that $M \cap N=H$ has maximal order. Let $L$ be a maximal subgroup of $G$ containing $N_{G}(H)$. Since $[G: H]$ is divisible only by $p$ or $q, L$ has index a power of $p$ or $q$. We may therefore assume that $(L, q) \in \psi$. Suppose now by way of contradiction that $L$ is Frobenius. Then $H \subseteq N \cap L=K$ where $K$ is a complement of $L$ by part (iii), Lemma 3. Now let $R$ be an $S_{r}$-subgroup of $G$ and also contained in $H$, for $r \in \pi(G)$. Then $\Omega_{1}(R)$ char $H, \Omega_{1}(R)$ char $K$.

Since $H$ and $K$ each is disjoint from its conjugates, we have

$$
N_{G}(H)=N_{G}\left(\Omega_{1}(R)\right)=N_{G}(K) .
$$

By lemma 3 (ii), $N_{G}(H)=N_{G}(K) \subseteq N$. By the same lemma 3 (ii) again, we conclude that $M$ is Frobenius contrary to the fact that $(M, N) \in \mathscr{I}$.

On the other hand, if $L$ is non-Frobenius, with $(L, q) \in \psi$, then $(N, L) \in$ $\Phi$. Since $H \subseteq N \cap L$, maximality of $H$ forces $H=N \cap L$. Since $N_{G}(H) \subseteq L$, it follows by Lemma 3 (ii) that $N$ is Frobenius - a contradiction. Thus $\mathscr{I}$ is empty.

(B). By (A) using Thompson's theorem ([2] p. 337) we obtain that a maximal subgroup of $G$ has in fact a nontrivial Fitting subgroup. By [1] lemma $4 \tau=\{p, q\}$. Next let $(U, q),(V, p) \in \psi$. By part (A) above we may assume that $U$ is a Frobenius group. By lemma 3 (ii) $U \cap V$ is a complement of $U$ and has odd order and so $U$ is soluble. Since $V=(U \cap V) Q$, where $Q$ 
is a Sylow $q$-subgroup of $G$, it follows by lemma 2 that $V$ is soluble also. Thus by the main theorem in [1], we conclude that

$$
G \simeq P S L(2,7) .
$$

\section{Acknowledgements}

This work is a part of my thesis accepted for the award of Ph.D degree by the University of London from Queen Mary College. I am indebted to Professor S. A. Huq and the referee for various helpful suggestions.

\section{References}

1. S. Adnan (1976), 'A characterization of PSL(2,7)', J. London Math. Soc. (2) 12.

2. D. Gorenstein (1969), Finite groups (Harper and Row).

3. B. Huppert (1963), Endliche Gruppen (Springer-Verlag, Berlin-Heidelberg-New York).

\section{Queen Mary College,}

Mile End Road,

London E. 1.

Present address: Faculty of Science, University of Khartoum, Khartoum, Sudan. 